\title{
Gamma-Ray Production in the Extended Halo of the Galaxy and Possible Implications for the Origin of Galactic Cosmic Rays
}

\author{
Ruo-Yu Liu ${ }^{1}$ (D), Huirong Yan ${ }^{1,2}$ (D), Xiang-Yu Wang ${ }^{3}$, Shi Shao ${ }^{4}$, and $\mathrm{Hui} \mathrm{Li}^{5}$ (1) \\ ${ }^{1}$ Deutsches Elektronen Synchrotron (DESY), Platanenallee 6, D-15738 Zeuthen, Germany; ruoyu.liu@desy.de, huirong.yan@desy.de \\ 2 Institut für Physik und Astronomie, Universität Potsdam, D-14476 Potsdam, Germany \\ ${ }^{3}$ School of Astronomy and Space Science, Nanjing University, Nanjing 210093, People's Republic of China \\ ${ }^{4}$ Institute for Computational Cosmology, Department of Physics, Durham University, South Road, Durham DH1 3LE, UK \\ ${ }^{5}$ Department of Physics, Kavli Institute for Astrophysics and Space Research, Massachusetts Institute of Technology, Cambridge, MA 02139, USA \\ Received 2018 May 11; revised 2018 November 29; accepted 2018 November 30; published 2019 January 21
}

\begin{abstract}
Various studies have implied the existence of a gaseous halo around the Galaxy extending out to $\sim 100 \mathrm{kpc}$. Galactic cosmic rays (CRs) that propagate to the halo, either by diffusion or by convection with the possibly existing large-scale Galactic wind, can interact with the gas therein and produce gamma-rays via proton-proton collision. We calculate the CR distribution in the halo and the gamma-ray flux, and explore the dependence of the result on model parameters such as diffusion coefficient, CR luminosity, and CR spectral index. We find that the current measurement of isotropic gamma-ray background (IGRB) at $\lesssim \mathrm{TeV}$ with the Fermi Large Area Telescope already approaches a level that can provide interesting constraints on the properties of Galactic CR (e.g., with CR luminosity $L_{\mathrm{CR}} \leqslant 10^{41} \mathrm{erg} \mathrm{s}^{-1}$ ). We also discuss the possibilities of the Fermi bubble and IceCube neutrinos originating from the proton-proton collision between CRs and gas in the halo, as well as the implication of our results for the baryon budget of the hot circumgalactic medium of our Galaxy. Given that the isotropic gamma-ray background is likely to be dominated by unresolved extragalactic sources, future telescopes may extract more individual sources from the IGRB, and hence put even more stringent restrictions on the relevant quantities (such as Galactic CR luminosity and baryon budget in the halo) in the presence of a turbulent halo that we consider.
\end{abstract}

Key words: cosmic rays - Galaxy: halo - gamma rays: diffuse background - neutrinos

\section{Introduction}

The question of how much cosmic-ray (CR) luminosity of our Galaxy is needed to maintain the observed CR flux was first raised by Ginzburg \& Syrovatskii (1964). The answer to this question not only provides a clue to the origin of Galactic CRs from the point of view of energetics that the sources can provide, but may also reflect the propagation nature of CRs inside the Galaxy and hence is also of great interest to other relevant fields such as the interstellar medium, plasma astrophysics, and so on. Based on the measurement of the local CR energy density and the so-called "grammage" (i.e., the amount of matter traversed by GCRs before reaching the Earth), which represents the propagation time of CRs, the CR luminosity of our Galaxy above $1 \mathrm{GeV}$ is usually found to be $3 \times 10^{40} \mathrm{erg} \mathrm{s}^{-1}<L_{\mathrm{CR}}<3 \times 10^{41} \mathrm{erg} \mathrm{s}^{-1}$ in the framework of the leaky-box model (Ginzburg \& Syrovatskii 1964; Drury et al. 1989; Berezinskii et al. 1990; Dogiel et al. 2002; Strong et al. 2010; O'C. Drury 2017), where the uncertainty is due to the statistical error in measurement and the selection of the CR propagation model. The estimated value of the CR luminosity provides an important clue to the species of CR accelerators and their efficiency.

CRs can be studied through the gamma-rays produced in the hadronuclear interaction or proton-proton collision (hereafter $p p$ collision) between CRs and diffuse baryonic material. This method has been widely used among the community, for example, to derive the $\mathrm{CR}$ distribution in the Galactic plane (e.g., Abdo et al. 2009) or as indicators of CR accelerators (e.g., Ackermann et al. 2013; HESS Collaboration et al. 2016). Interestingly, recent observations of ion absorption lines against background quasars (e.g., Nicastro et al. 2002;
Rasmussen et al. 2003; Miller \& Bregman 2013; Fang et al. 2015; Zheng et al. 2017) and emission lines (e.g., Henley \& Shelton 2012, 2013; Miller \& Bregman 2015) at high Galactic latitudes along different lines of sight suggest the existence of a hot baryon gas halo surrounding the Galaxy, which is also known as the circumgalactic medium (CGM). The existence of the CGM is also supported by various indirect observations (e.g., Stanimirović et al. 2002; Fox et al. 2005; Grcevich \& Putman 2009; Putman et al. 2011). The total mass of the CGM is inferred to be several times $10^{10} M_{\odot}$ within the virial radius $(250 \mathrm{kpc})$ of the Galaxy. It serves as a target of baryons and can interact with CRs that escaped our Galaxy in the past via $p p$ collision and give rise to gamma-ray photons. The gamma-ray photons produced at high Galactic latitudes may/will contribute to the isotropic gamma-ray background (IGRB) as measured by various instruments such as the $S A S-2$ satellite (Fichtel et al. 1975, 1978), EGRET on board the Compton Observatory (Sreekumar et al. 1998; Strong et al. 2004), and the Fermi Large Area Telescope (Fermi-LAT; Abdo et al. 2010) up to $100 \mathrm{GeV}$.

The $p p$ collision between the gas and CRs including the consequent production of gamma-rays in the Galactic halo has been considered by various authors (Stecker \& Jones 1977; De Paolis et al. 2000; Feldmann et al. 2013; Ahlers \& Murase 2014; Taylor et al. 2014). Recently, Fermi-LAT updated the spectrum measurement on IGRB up to to $820 \mathrm{GeV}$ (Ackermann et al. 2015). Extrapolating the prediction of earlier works to this sub-TeV energy range and comparing them to the new FermiLAT measurement allows some of the physical setups/ parameter ranges considered by the authors to be ruled out. More recently, Kalashev \& Troitsky (2016) addressed the 
sub-TeV measurement of the extragalactic gamma-ray background (EGB), which also includes the extragalactic pointsource contribution in addition to the IGRB. Although their result is consistent with the sub-TeV EGB flux, it is likely to be in tension with the sub-TeV IGRB flux.

In this work, we will study gamma-rays produced in the interactions between the CRs and the gas in the halo, and use the results to put constraints on model parameters such as the CR luminosity of the Galaxy. We take into account diffusion in turbulent halo and the convection by the large-scale Galactic wind that might exist and the subsequent adiabatic cooling of CRs in the wind, which were ignored in some previous studies. We will obtain constraints, which are independent of direct measurements on CRs, on various properties of Galactic CRs as well as the diffusion coefficient in the halo and the mass of CGM from the IGRB flux at $\lesssim \mathrm{TeV}$ energies measured by Fermi-LAT.

This paper is organized as follows. We calculate the CR distribution and the gamma-ray production in the halo in Section 2. In Section 3, the results are presented and the constraint on the Galactic CR luminosity is discussed. We discuss the anisotropy of the gamma-ray intensity, the neutrino emission, and the baryon budget of the Galaxy in Section 4, and summarize the work in Section 5.

\section{Cosmic-Ray Propagation and Gamma-Ray Production}

To calculate the gamma-ray flux originating from the halo, we need the distribution of CRs and gas in the halo. In this section, we first study the propagation and evolution of CRs in the halo. In our model, we consider $\mathrm{CR}$ propagation by diffusion, convection by the Galactic wind, and the cooling of the particles. Then, we use the CR and gas distribution in the CGM to calculate the gamma-ray emissivity in the halo and the total gamma-ray flux at the Sun's position in the Galaxy, for comparison with observations.

The transport of CRs injected from a point source is regulated by the equation (Berezinsky \& Gazizov 2006)

$$
\begin{aligned}
& \frac{\partial n}{\partial t}+\boldsymbol{v}_{w} \nabla_{\boldsymbol{r}} n-D(E) \nabla_{\boldsymbol{r}}^{2} n-\frac{\partial}{\partial E}[b(E, t) n] \\
& \quad=Q(E, t) \delta^{3}\left(\boldsymbol{r}-\boldsymbol{r}_{g}\right)
\end{aligned}
$$

where $n(\boldsymbol{r}, E, t)$ is the differential density of CRs at time $t$ and at $\boldsymbol{r}=(x, y, z)$, which are the Cartesian coordinates of a certain position in space measured in the comoving frame. $\boldsymbol{r}_{g}$ is the coordinate of the point source. We define the coordinates of the Galactic center (GC) and the Earth to be $\boldsymbol{r}_{C}=(0,0,0)$ and $\boldsymbol{r}_{E}=(8.5 \mathrm{kpc}, 0,0)$, respectively, and $t=0$ at the present time.

The second term represents the effect of the convection by a large-scale Galactic wind, which could be launched by the pressure gradient of CRs (e.g., Breitschwerdt et al. 1991; Zirakashvili et al. 1996; Hanasz et al. 2013; Salem \& Bryan 2014). The Galactic wind may be alternatively launched by supernova explosions, as studied in Dubois \& Teyssier (2008; Sarkar et al. 2015; Fielding et al. 2017). $\boldsymbol{v}_{w}$ is the velocity of the wind, the value of which is found to increase with distance from the Galactic plane. According to the reference model in Zirakashvili et al. (1996) or the fiducial model in Recchia et al. (2016), the wind velocity finally approaches an asymptotic value of $\sim 300 \mathrm{~km} \mathrm{~s}^{-1}$ at $\sim 100 \mathrm{kpc}$. The wind velocity in other studies is also a few times $100 \mathrm{~km} \mathrm{~s}^{-1}$ at $\sim 100 \mathrm{kpc}$. Thus, we take $v_{w}=300 \mathrm{~km} \mathrm{~s}^{-1}$ as the fiducial value for the wind velocity in the calculation and assume it to be constant throughout the halo. Such a treatment will overestimate the adiabatic cooling of CRs at smaller radius (see the discussion in Section 3).

The third term considers the CR diffusion with the diffusion coefficient $D(E)=c l_{\mathrm{mfp}}(E) / 3$, where $l_{\mathrm{mfp}}$ is the mean free path of CRs. For simplicity, the diffusion coefficient is assumed to be independent of both time and space. The diffusion coefficient in the halo is an important parameter for our calculation but, unfortunately, there are still some uncertainties. On one hand, the turbulence might be quite weak and hence the diffusion coefficient could be large in the extended halo, if the injection of turbulence into the halo originates from the Galactic plane. On the other hand, CRs may also stream down their pressure gradient by scattering off self-excited Alfvén waves (e.g., Kulsrud \& Pearce 1969; Skilling 1971). The selfregulated transport could lead to a small diffusion efficiency if the CR density is sufficiently high and the wave damping process is weak. As pointed out in earlier studies (Yan \& Lazarian 2002; Farmer \& Goldreich 2004), the streaming instability may be suppressed at high energies such as multi$\mathrm{TeV}$, with which we are mainly concerned, due to a low CR density at such high energies and also due to the wave damping by the nonlinear Landau damping in the hot plasma. In this work, we do not incorporate the influence of the streaming instability in the calculation; instead, we follow the mean free path of CRs calculated by Yan \& Lazarian (2008), which is based on the current understanding of Galactic turbulence and calculated with nonlinear theory to calculate the diffusion coefficient for the entire halo (as is shown in panel a of Figure 1). We note that although $\mathrm{CR}$ convection by the Galactic wind and diffusion may be somehow related (e.g., the streaming instability of CRs), as a phenomenological study, we treat them (i.e., $v_{w}$ and $D$ ) as independent parameters in this work. We will discuss in detail the dependence of our result on the diffusion coefficient below.

The last term on the left-hand side describes the effect of continuous energy loss, where

$$
\begin{aligned}
& b(E, r, t)=-\frac{d E}{d t}=\kappa \sigma_{p p}(E) n_{g}(r) c E+\left(v_{w} / r\right) E \\
& =6 \times 10^{-7}\left(\frac{n_{g}}{10^{-3} \mathrm{~cm}^{-3}}\right)\left(\frac{E}{10^{12} \mathrm{eV}}\right) \mathrm{eV} \mathrm{s}^{-1} \\
& \quad+10^{-3}\left(\frac{v_{w}}{300 \mathrm{~km} \mathrm{~s}^{-1}}\right)\left(\frac{r}{10 \mathrm{kpc}}\right)^{-1}\left(\frac{E}{10^{12} \mathrm{eV}}\right) \mathrm{eV} \mathrm{s}^{-1}
\end{aligned}
$$

is the energy loss rate due to $p p$ collision and the adiabatic expansion of the wind. $\kappa \simeq 0.5$ is the inelasticity of the $p p$ collision, and $\sigma_{p p}$ is the cross-section, which is about $40 \mathrm{mb}$ at several TeV (Kelner et al. 2006). $n_{g}(r)$ is the gas density profile in the halo and is important to gamma-ray production because it is proportional to the $p p$ collision rate. Miller \& Bregman (2015) analyzed the O VII and O VIII emission lines considering different plasma conditions in the halo, and obtained a total mass of the hot gas within the virial radius ranging from $2.7 \times 10^{10}$ to $9.1 \times 10^{10} M_{\odot}$ under the best-fit parameters. We then employ the so-called $\beta$-model in the form of $n_{g}(r)=n_{0}(r / 1 \mathrm{kpc})^{-3 \beta}$, with two sets of parameters that correspond to the lower bound of the total gas mass, i.e., 

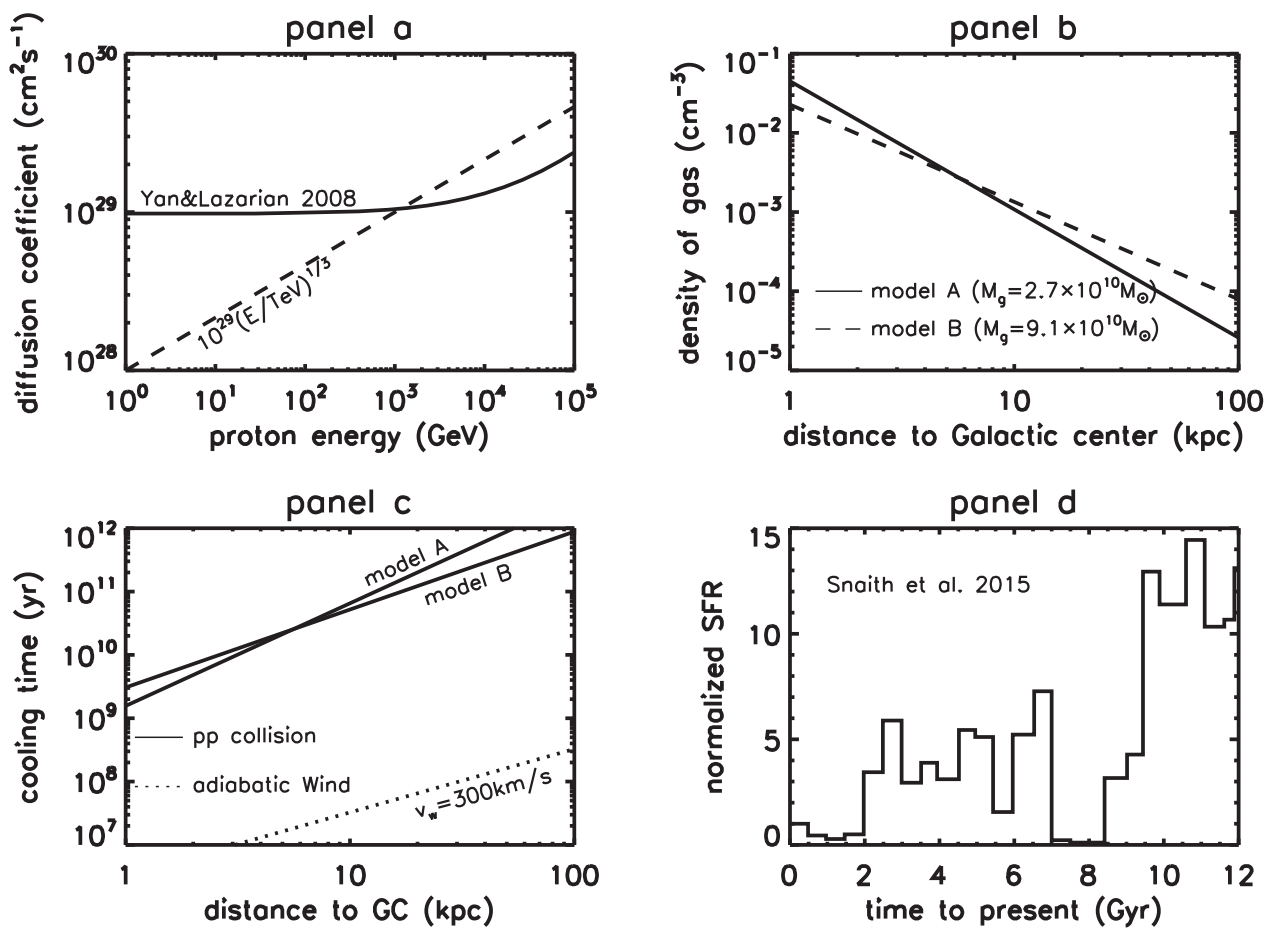

Figure 1. Model parameters and basic properties. Top left (panel a): diffusion coefficient in the halo obtained by Yan \& Lazarian (2008); the usually adopted form of diffusion coefficient in the Galactic disk $D(E)=10^{29}(E / \mathrm{TeV})^{1 / 3} \mathrm{~cm}^{2} \mathrm{~s}^{-1}$ is also plotted for reference. Top right (panel b): density profile of hot gas in the halo; bottom left (panel c): cooling timescale due to $p p$ collision (solid lines) and adiabatic loss due to the expansion of the Galactic wind (dotted line); bottom right (panel d): SFH obtained by Snaith et al. (2015), normalized to 1 at present day.

$n_{0}=0.045 \mathrm{~cm}^{-3}$ and $\beta=0.54$ (hereafter, model $\mathrm{A}$ and the fiducial case), and the upper bound of the total gas mass, i.e., $n_{0}=0.023 \mathrm{~cm}^{-3}$ and $\beta=0.41$ (hereafter, model $\mathrm{B}$ ) obtained in their work (see panel b in Figure 1). Note that the gas density depends on the metallicity, which is assumed to be $Z=0.3 Z_{\odot}$, where $Z_{\odot}$ is the solar metallicity. This assumption is consistent with the residual pulsar dispersion measure toward the Large Magellanic Cloud (LMC; Miller \& Bregman 2015) and simulations of the Galactic corona (Toft et al. 2002; Cen \& Ostriker 2006; Cen 2012). Given a density of $10^{-3}-10^{-5} \mathrm{~cm}^{-3}$ in the halo, we expect the energy loss to be dominated by the adiabatic loss due to the expansion of the Galactic wind. We plot the $\mathrm{CR}$ cooling time, calculated as $E / b(E, t, r)$ for different cooling mechanisms in panel c of Figure 1.

The term on the right-hand side of Equation (1) represents the injection of CRs, which are assumed to consist of pure protons in this work, from a point source located at $\boldsymbol{r}_{g}$. We assume that the injection rate at time $t$ for CRs with energy $E$ follows the form

$$
\begin{aligned}
& Q(E, t)=S(t) Q_{0}(E)=S(t) N_{0}(E / 1 \mathrm{GeV})^{-p} \\
& \quad \times \exp \left(-E / E_{\max }\right)
\end{aligned}
$$

where $S(t)$ describes the CR injection history. $p$ is the slope of the injection spectrum. The locally observed slope of the CR spectrum is about 2.7 , implying an injection slope of 2.4 given a diffusion coefficient $D(E) \propto E^{1 / 3}$ in the ISM (Aguilar et al. 2016). On the other hand, a recent gamma-ray observation of various molecular clouds (Neronov et al. 2017) and diffuse gamma-ray emission from the inner Galaxy (Yang et al. 2016) suggest the slope of the CR spectrum to be $\sim 2.3-2.5$, implying an injection slope of 2.0-2.2. Thus, we take $p=2.2$ as a fiducial value and explore the influence of $p$ in the range of 2.0-2.4. $E_{p, \max }$ represents the cutoff energy in the injection spectrum, and we assume $E_{p, \max }=10^{15} \mathrm{eV}$, which is comparable to the break in the measured CR spectrum (or the so-called "knee"). Since CRs are generally believed to be accelerated via strong shocks of supernova remnants, we assume that the $\mathrm{CR}$ injection history follows roughly the star formation history (SFH) of the Galaxy. Snaith et al. (2015) derived the SFH of our Galaxy by fitting their chemical evolution model to a large sample of stellar abundances, and we adopt this one for $S(t)$, which is shown in Figure 1(d). Our adopted SFH is also consistent in general with the SFH for MilkyWay-sized galaxies derived from abundance matching techniques (Behroozi et al. 2013). Given the present CR luminosity $L_{\mathrm{CR}, 0}$ and assuming CRs are injected homogeneously from the Galactic plane with a radius of $R_{\mathrm{Gal}}=15 \mathrm{kpc}$ and a negligible thickness (i.e., $\boldsymbol{r}_{g}=\left(x_{g}, y_{g}, 0\right)$ with $\sqrt{x_{g}^{2}+y_{g}^{2}}<15 \mathrm{kpc}$ ), we can find $N_{0}$ from $\pi R_{\mathrm{Gal}}^{2} \int_{1 \mathrm{GeV}}^{\infty} E Q_{0}(E) d E=L_{\mathrm{CR}, 0}$. We note that the source of CRs from the Galactic plane is probably not homogeneously distributed, and the disk size also evolves with redshift. However, because we are concerned with the CR distribution in a $100 \mathrm{kpc}$ scale halo, the dependence on the distribution of CR sources in the Galactic plane or on the disk size is not significant. The input model parameters and their values in the fiducial case are summarized in Table. 1.

Following the method of Berezinsky \& Gazizov (2006) to solve Equation (1) in Fourier space and transform it back to real space, we obtain the analytical solution of Equation (1) for a 
Table 1

Input Parameters in the Model

\begin{tabular}{clc}
\hline \hline Parameters & Descriptions & Values in the fiducial case \\
\hline$L_{\mathrm{CR}, 0}$ & Galactic CR luminosity at the present time & $10^{41} \mathrm{erg} \mathrm{s}^{-1}$ \\
$p$ & Spectral index of CRs at injection & 2.2 \\
$E_{p, \text { max }}$ & Cutoff energy in the CR injection spectrum & $10^{15} \mathrm{eV}$ \\
$D(E)$ & Diffusion coefficient of CRs of energy $E$ in the halo & Following Yan \& Lazarian (2008) \\
$v_{w}$ & Velocity of the large-scale Galactic wind & $300 \mathrm{~km} \mathrm{~s}^{-1}$ \\
$n_{0}$ & Halo gas density normalized at $1 \mathrm{kpc}$ from the Galactic center & $0.045 \mathrm{~cm}^{-3}\left(\mathrm{Miller} \&\right.$ Bregman 2015) $_{\beta}$ \\
$S(t)$ & Slope of the density profile & 0.54 (Miller \& Bregman 2015) \\
& Normalized CR injection history with $S(0)=1$ (the present-time value) & Following Snaith et al. (2015) (see panel d of Figure 1)
\end{tabular}

point source located at $\boldsymbol{r}_{g}$,

$$
\begin{aligned}
n\left(t, \boldsymbol{r}, E ; \boldsymbol{r}_{g}\right) & =\frac{\pi^{3 / 2}}{(2 \pi)^{3}} \int_{t_{g}}^{t} d t^{\prime} Q\left(\mathcal{E}^{\prime}, t^{\prime}\right) \\
& \times \frac{\exp \left[-\left(\boldsymbol{r}-\boldsymbol{r}_{\boldsymbol{g}}-\boldsymbol{s}\right)^{2} / 4 \lambda\left(E, t^{\prime}\right)\right]}{\lambda\left(E, t^{\prime}\right)^{3 / 2}} \\
& \times \exp \left[\int_{t^{\prime}}^{t} d t^{\prime \prime} \frac{\partial b\left(\mathcal{E}^{\prime \prime}, t\right)}{\partial \mathcal{E}^{\prime \prime}}\right],
\end{aligned}
$$

where $s=\int_{t^{\prime}}^{t} v d t^{\prime \prime}$ and $\lambda\left(E, t^{\prime}\right)=\int_{t^{\prime}}^{t} D\left(\mathcal{E}^{\prime \prime}\right) d t^{\prime \prime}$. Here, $\mathcal{E}\left(t^{\prime \prime}, E\right)$ or $\mathcal{E}\left(t^{\prime}, E\right)$ means the energy of a $\mathrm{CR}$ at time $t^{\prime \prime}$ or $t^{\prime}$, which has energy $E$ at the present time. The above solution is the CR density from a point source at $\boldsymbol{r}_{g}$, so we need to further integrate over the Galactic plane in order to obtain the contribution from the whole Galaxy. We set the earliest injection of CRs to have started at 12 Gyr ago (i.e., $t_{g}=-12 \mathrm{Gyr}$ ), which corresponds to a redshift of 4 . Since we assume that $\mathrm{CR}$ injection history follows the SFH of the Galaxy, our selection of $t_{g}$ includes most CRs injected in the history (see Figure 1(d)). Besides, CRs injected at earlier time have propagated to a quite large distance, which is $\sim 2 \sqrt{D t} \simeq 120 \mathrm{kpc}$ for diffusion and $\sim v_{w} t \sim 3.6 \mathrm{Mpc}$ for convection given a time $t=12 \mathrm{Gyr}$, and hence have small contribution to gamma-rays. Thus, considering earlier injection barely changes the results but increases the calculation time. Then, we can obtain the CR density distribution in the halo at present time $(t=0)$,

$$
N(\boldsymbol{r}, E)=\iint n\left(t=0, \boldsymbol{r}, E ; \boldsymbol{r}_{g}\right) d x_{g} d y_{g} .
$$

In Figure 2, we show the distribution of $1 \mathrm{TeV} C \mathrm{CR}$ energy density along the $z$-direction, i.e., the axis perpendicular to the Galactic plane passing through the $\mathrm{GC}$ at $(0,0,0)$ (black curves), the one passing through the Earth at (8.5 kpc, 0, 0) (blue curves), and the one passing through the edge of the Galactic plane at $(15 \mathrm{kpc}, 0,0)$ (red curves). Compared to the pure diffusion case, the presence of a Galactic wind will transport CRs to farther distances within the same amount of time. The adiabatic cooling of CRs in the expanding wind also leads to an extra energy loss of CRs especially at small Galactocentric radius. As a result, the CR density within $100 \mathrm{kpc}$ in the case without wind is higher than that in the case with wind.

Once we have the CR distribution and gas distribution in the halo, we can calculate the gamma-ray emissivity $\left(\mathrm{GeV} \mathrm{cm}^{-3} \mathrm{~s}^{-1}\right)$ at $\boldsymbol{r}$, which is denoted by

$$
\begin{aligned}
J_{\gamma}\left(E_{\gamma}, \boldsymbol{r}\right) & \equiv \frac{d N_{\gamma}}{d E_{\gamma} d t} \\
& =c n_{\mathrm{g}}(\boldsymbol{r}) \int_{E_{\gamma}}^{\infty} \sigma_{p p} N(\boldsymbol{r}, E) F_{\gamma}\left(\frac{E_{\gamma}}{E}, E\right) \\
\frac{d E}{E}\left(E_{\gamma}, \boldsymbol{r}\right) & \equiv \frac{d N_{\gamma}}{d E_{\gamma} d t} \\
& =c n_{\mathrm{g}}(\boldsymbol{r}) \int_{E_{\gamma}}^{\infty} \sigma_{p p} N(\boldsymbol{r}, E) F_{\gamma}\left(\frac{E_{\gamma}}{E}, E\right) \frac{d E}{E},
\end{aligned}
$$

following the semi-analytic method developed by Kelner et al. (2006), where $F_{\gamma}$ is the spectrum of the secondary gamma-ray in a single collision. ${ }^{6}$ The total gamma-ray flux average over the solid angle at Earth can then be given by

$$
\begin{aligned}
\Phi_{\gamma}\left(E_{\gamma}\right)= & \frac{1}{4 \pi\left(1-\cos 70^{\circ}\right)} \iiint d x d y d z \frac{J_{\gamma}\left(E_{\gamma}, \boldsymbol{r}\right)}{4 \pi\left(\boldsymbol{r}-\boldsymbol{r}_{E}\right)^{2}} \\
& \times\left[1-\theta(3 \mathrm{kpc}-|z|) \theta\left(15 \mathrm{kpc}-\sqrt{x^{2}+y^{2}}\right)\right] \\
& \times \theta\left(\sin ^{-1} \frac{|z|}{\left|\boldsymbol{r}-\boldsymbol{r}_{E}\right|}-20^{\circ}\right),
\end{aligned}
$$

where $\theta(x)$ is the Heaviside function. The term in the square brackets is to subtract the emission inside the Galaxy, which is regarded as a cylinder with a radius of $15 \mathrm{kpc}$ and a half-height of $3 \mathrm{kpc}$ above and below the Galactic plane, while the last Heaviside function is to subtract the low-latitude (Galactic latitude $|b|<20^{\circ}$ ) emission following the measurement of IGRB by Fermi-LAT (Ackermann et al. 2015). That is also the reason that the term $1-\cos 70^{\circ}$ appears in the denominator of the prefactor of the integration when we average the total flux over the solid angle. Note that given a diffusion coefficient of $10^{29} \mathrm{~cm}^{2} \mathrm{~s}^{-1}$, we expect CRs can diffuse to a distance of $2 \sqrt{D \Delta t} \simeq 100 \mathrm{kpc}$ with a propagation time of $\Delta t=12 \mathrm{Gyr}$, so we only sum up the emission out to $|\boldsymbol{r}|=100 \mathrm{kpc}$. Although CRs can travel to a much larger distance in the presence of a Galactic wind, the contribution from larger distances is subdominant because both the CR density and the gas density are very low there.

In the calculation of gamma-ray flux, we do not consider the contribution of secondary electrons produced in the $p p$ collision

\footnotetext{
6 See Section IV.A of Kelner et al. (2006), which is based on the SIBYLL code (Fletcher et al. 1994).
} 


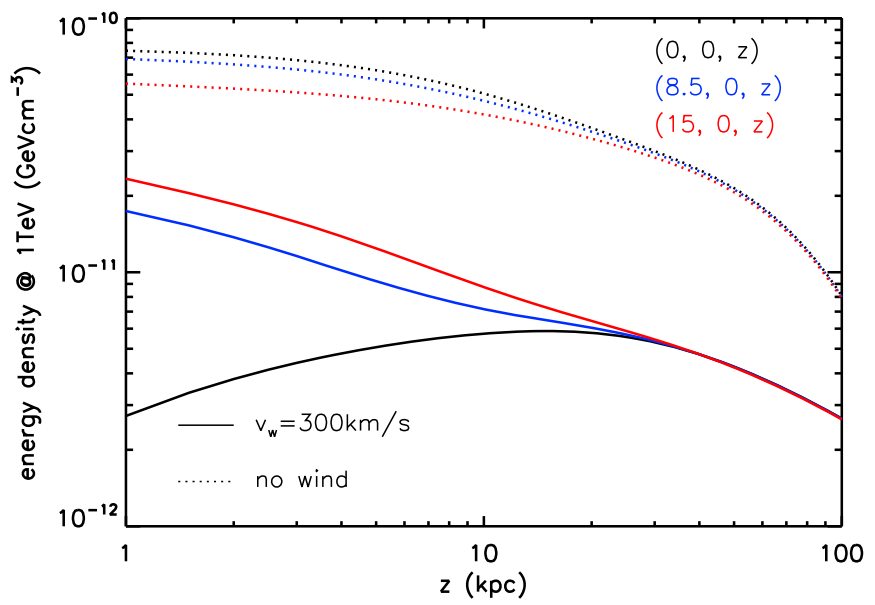

Figure 2. Distributions of CR energy density at the present time at $1 \mathrm{TeV}$ along the axes perpendicular to the Galactic plane, passing through the GC (i.e., point $(0,0,0)$, black curves), Earth (i.e., point $(8.5 \mathrm{kpc}, 0,0)$, blue curves), and the edge of the Galactic plane (i.e., point $(15 \mathrm{kpc}, 0,0)$, red curves). The solid and dotted curves, respectively, represent the cases with the large-scale radial Galactic wind of $v_{w}=300 \mathrm{~km} \mathrm{~s}^{-1}$ present and without the wind present.

via the decay of charged pions, which can emit gamma-rays by inverse Compton scattering off CMB photons. This is because, first, the electron production rate is about half of the gamma-ray production rate in the $p p$ collision of the same parent protons. Second, to emit $\mathrm{TeV}$ photons via inverse Compton scattering off CMB photons, which are produced by about $200 \mathrm{TeV}$ protons in $p p$ collisions, the electron energy needs to be about $10 \mathrm{TeV}$, while the required energy of protons that produce $\mathrm{TeV}$ photons is about $10 \mathrm{TeV}$. Thus, the gamma-ray flux from the secondary electrons is subordinate unless the injection spectrum is hard (i.e., $p<2$ ). We also ignore the contribution from electrons produced in the electromagnetic cascades initiated by the high-energy photons propagating in the CMB and infrared photon field. This is because the mean free path of $20 \mathrm{TeV}$ photons that produce $10 \mathrm{TeV}$ electron/positron pairs is about $100 \mathrm{Mpc}$ (e.g., Coppi \& Aharonian 1997), which is much larger than the size of the Galactic halo.

\section{Gamma-Ray Flux and the Constraint on the Galactic CR Luminosity}

With the obtained gamma-ray flux, we can put a constraint on Galactic CR luminosity by requiring the gamma-ray flux not to overshoot the observed IGRB flux. On the other hand, the result also depends on parameters other than CR luminosity. Thus, we will explore the influence of these parameters, with a particular focus on the diffusion coefficient.

The measured IGRB flux by Fermi-LAT is shown with black circles in Figure 3. The spectrum approximately follows a power law of index of -2.3 and steepens around $100 \mathrm{GeV}$. The flux of the highest energy bin at (580-820) GeV with a center energy of $700 \mathrm{GeV}$ is consistent with zero, and hence an $85 \%$ C.L. upper limit of $4 \times 10^{-9} \mathrm{GeV} \mathrm{cm}^{-2} \mathrm{~s}^{-1} \mathrm{sr}^{-1}$ is obtained (corresponding to the upper bound of the $1 \sigma$ uncertainty interval) based on the foreground model $\mathrm{A}$ in Ackermann et al. (2015). Such an upper limit is supposedly to give the most stringent constraint on the model parameters. Note that the foreground model is important to the IGRB flux but it will not affect our result significantly. The upper limit flux will be increased by about a factor of $\sim 1.5$ under other foreground models.

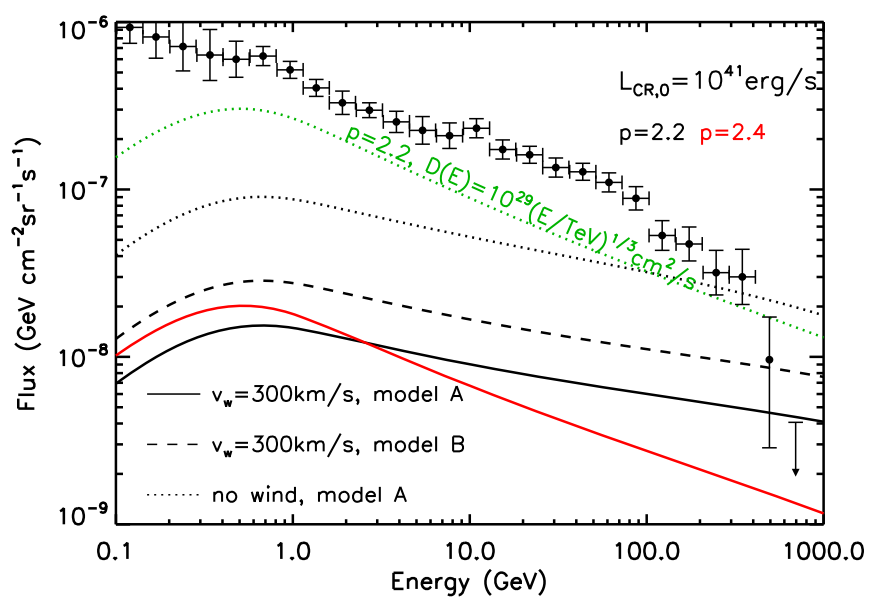

Figure 3. Predicted gamma-ray flux from the extended halo of the Galaxy. The solid and dashed curves represent the gamma-ray flux considering a Galactic wind of constant radial velocity of $300 \mathrm{~km} \mathrm{~s}^{-1}$, for the gas density profile model A $\left(M_{g}=2.7 \times 10^{10} M_{\odot}\right)$ and model B $\left(M_{g}=9.1 \times 10^{10} M_{\odot}\right)$, respectively. The dotted curve represents the case of no wind for model A. The injection spectral index of CRs is 2.2 for black curves and 2.4 for the red curve. The green dotted curve represents the flux calculated by adopting the diffusion coefficient of the disk for the entire halo. In all cases shown here, the CR luminosity is $10^{41} \mathrm{erg} \mathrm{s}^{-1}$. Filled circles with error bars are IGRB data measured by Fermi-LAT (Ackermann et al. 2015). The upper limit at the highest energy bin (580-820 GeV) is shown with the downward arrow.

\subsection{Comparison of Expected Gamma-Ray Fluxes in Different Cases}

Along with the IGRB data, we compare the predicted gamma-ray flux from the halo in the fiducial case to those with variations in some parameters in Figure 3. The effect of the gas content on the halo can be seen by comparing the black solid curve (the fiducial case) and black dashed curve. The gas density model $\mathrm{B}\left(M_{g}=9.1 \times 10^{10} M_{\odot}\right)$ provides more target atoms for $p p$ collision than the gas density model A $\left(M_{g}=2.7 \times 10^{10} M_{\odot}\right)$, by a factor of $\gtrsim 3$. As a result, the gamma-ray flux in the former case is naturally higher than that in the latter case by about a factor of 2 .

For the fiducial diffusion coefficient and a wind speed of $300 \mathrm{~km} \mathrm{~s}^{-1}$, convection dominates $\mathrm{CR}$ transportation at $r \gg 4\left(D / 10^{29} \mathrm{~cm}^{2} \mathrm{~s}^{-1}\right)\left(v_{w} / 300 \mathrm{~km} \mathrm{~s}^{-1}\right)^{-1} \mathrm{kpc}$. Without the Galactic wind, the CR density will be higher and increase the gamma-ray flux (see Figure 2). In previous studies (Feldmann et al. 2013; Kalashev \& Troitsky 2016), the authors did not consider the Galactic wind. The gamma-ray flux in our "no wind" case is comparable to their results, although they adopted a higher gas density while we adopted a smaller diffusion coefficient.

The gamma-ray flux is sensitive to the injection spectrum of CRs. Comparing the black solid curve to the red solid curve, we can see that the flux at the highest energy bin decreases by a factor of 3 when $p$ changes from 2.2 to 2.4. Also, it is obvious that the flux should be proportional to the total CR luminosity at the present time $L_{\mathrm{CR}, 0}$.

Note that the diffusion coefficient is an important parameter to the result as we mentioned earlier, since the flux is roughly proportional to $1 / D$ in the region where diffusion dominates the CR transportation. For reference, we show the gammaray flux by applying the diffusion coefficient $D(E)=$ $10^{29}(E / 1 \mathrm{TeV})^{1 / 3} \mathrm{~cm}^{2} \mathrm{~s}^{-1}$ without wind to the entire halo (green dotted curve in Figure 3). A comparable gamma-ray flux at $\lesssim \mathrm{TeV}$ is obtained since the diffusion coefficient for 
$\lesssim 10 \mathrm{TeV}$ CRs is similar. The diffusion coefficient in this case for CRs with $E<10 \mathrm{GeV}$ is one order of magnitude smaller than that of Yan \& Lazarian (2008). As a result, the $\mathrm{GeV}$ gamma-ray emission in this case is higher.

\subsection{Parameter Space Exploration}

We note that there are some uncertainties in the model parameters for CR transportation. First, our assumption of a constant radial Galactic wind may underestimate the gammaray flux. In fact, whether a large-scale Galactic wind can be launched is still uncertain (e.g., Dubois \& Teyssier 2008). On the other hand, the wind velocity is probably perpendicular to the Galactic plane at $z \lesssim 15 \mathrm{kpc}$ rather than radial so that CRs will not suffer adiabatic loss (although the wind may open to a spherical shape at large Galactocentric radius; see Zirakashvili et al. 1996; Recchia et al. 2016). Also, the profile of the wind speed is unlikely to be a constant. According to the calculations in previous publications (e.g., Breitschwerdt et al. 1991; Zirakashvili et al. 1996; Recchia et al. 2016), the wind speed varies with the distance from the Galactic plane, and in most regions, the wind speed is smaller than $300 \mathrm{~km} \mathrm{~s}^{-1}$, especially near the Galactic plane (see also Taylor \& Giacinti 2017). In addition, cosmological simulations suggest a weaker Galactic wind at earlier times (Vogelsberger et al. 2013; Muratov et al. 2015). Apparently, our assumption of Galactic wind leads to an unrealistically efficient adiabatic loss of CRs at small Galactocentric radius and an overly fast transport of CRs to the outer halo by the wind. Nevertheless, the assumption of a constant wind enables a simple analytic solution to the CR transport equation (Equation (4)). Thus, instead of employing a more realistic Galactic wind, we propose that the gamma-ray fluxes obtained in the case of no Galactic wind and in the case of a constant wind velocity $v_{w}=300 \mathrm{~km} \mathrm{~s}^{-1}$ represent, respectively, an upper bound and a lower bound for the gamma-ray flux in the realistic Galactic wind case.

Second, the employed benchmark diffusion coefficient (Yan \& Lazarian 2008) is calculated based on the condition of turbulence in the inner halo $\left(r \lesssim 10 \mathrm{kpc}\right.$, with $n \sim 10^{-3} \mathrm{~cm}^{-3}$, $B \sim \mu \mathrm{G}, T \sim 10^{6} \mathrm{~K}$, turbulence injection scale $L \sim 100 \mathrm{pc}$ ) and results in a diffusion coefficient of $\gtrsim 10^{29} \mathrm{~cm}^{2} \mathrm{~s}^{-1}$ for CRs of $\lesssim 10 \mathrm{TeV}$, which is close to the diffusion coefficient in the Galactic disk. This is consistent with results of previous studies on the $\lesssim 10 \mathrm{kpc}$ radio halo/extended disks of our Galaxy and nearby spiral galaxies (e.g., Orlando \& Strong 2013; Mulcahy et al. 2016; Heesen et al. 2018). However, the diffusion coefficient in the extended halo or the outer halo is less known, and it is not necessarily the same as that in the inner halo. Thus, in the remainder of this section, we focus on exploring how the diffusion coefficient affects the constraint on CR luminosity.

Since our treatment for CR transportation in this work is only applicable to a spatially independent diffusion coefficient, a homogeneous diffusion coefficient is still adopted for the entire halo. We then assume $D(E)=D(1 \mathrm{TeV})(E / 1 \mathrm{TeV})^{1 / 3}$ with $D(1 \mathrm{TeV})$ being a free parameter, and calculate the gamma-ray flux with different spectral indexes of CRs at injection $(p)$ and obtain an upper limit for the CR luminosity at present time by normalizing the predicted gamma-ray flux to the IGRB upper limit at the highest energy bin of 500-820 GeV with the central energy $700 \mathrm{GeV}$. The results are shown in Figure 4. For each curve, the left side is the allowed parameter space while the right side is the excluded one. Basically, the smaller the diffusion coefficient is, the more stringent the
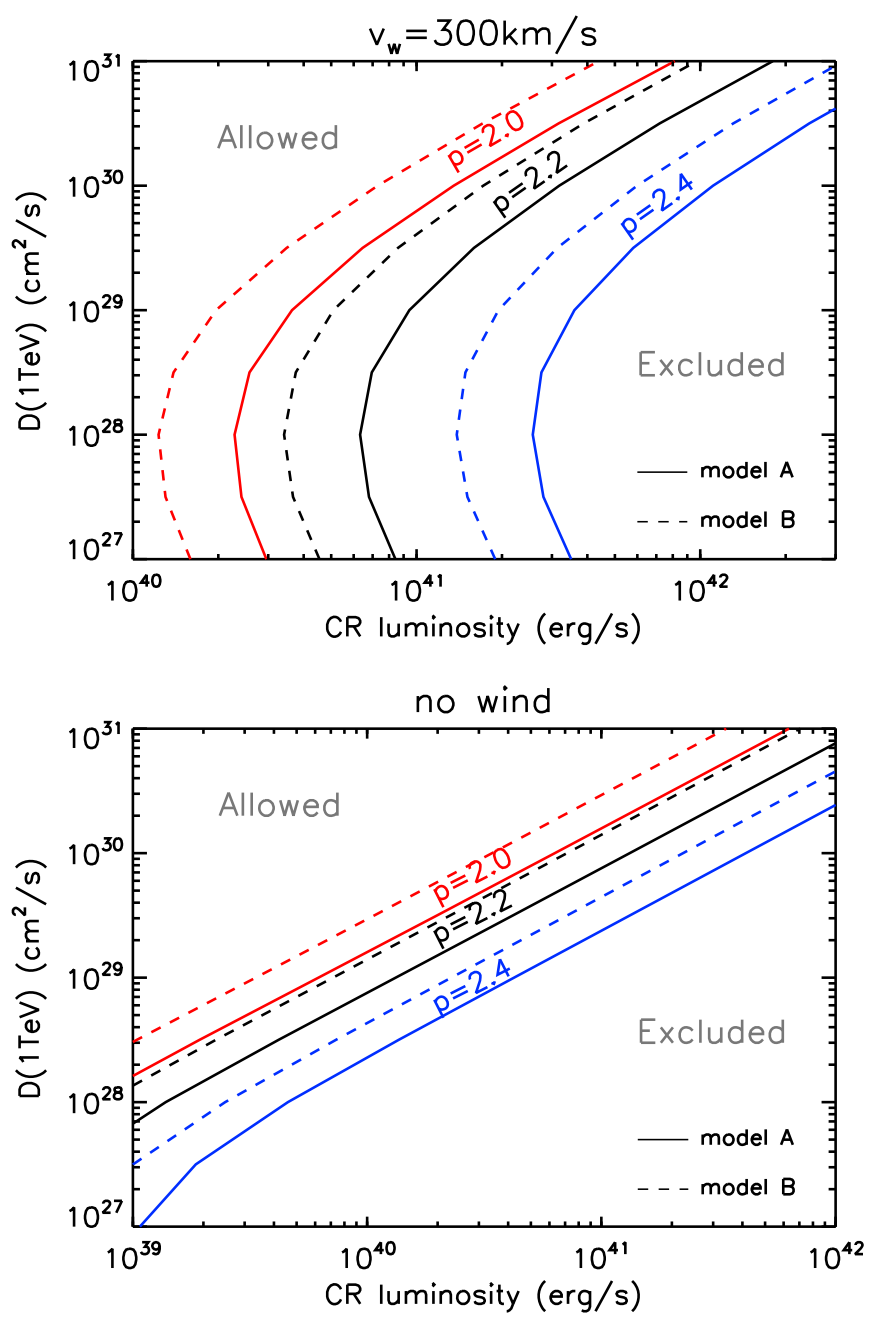

Figure 4. The maximum $C R$ luminosity that will not overshoot the IGRB upper limit at $700 \mathrm{GeV}$ as a function of the diffusion coefficient in the halo. The diffusion coefficient is assumed to take the form of $D(E)=$ $D(1 \mathrm{TeV})(E / 1 \mathrm{TeV})^{1 / 3}$. Solid curves represent the results with model A of the halo gas density while dashed curves represent those with model B. Three different injection spectral indexes are considered (red curves for $p=2.0$, black curves for $p=2.2$, and blue curves for $p=2.4$ ). Upper panel: a radial Galactic wind with a constant speed of $v_{w}=300 \mathrm{~km} \mathrm{~s}^{-1}$ is assumed. Lower panel: no wind is present.

constraint on the CR luminosity will be. The turnovers in the curves when the diffusion coefficient is small are caused by two reasons: first, CRs are trapped in the wind when the diffusion coefficient is small so adiabatic losses of CRs are severe; second, even if there is no wind, because we do not count the gammarays produced at small latitude $\left(|b|<20^{\circ}\right)$ and inside the Galaxy (which is considered as a cylinder with a radius of $15 \mathrm{kpc}$ and a half-height of $3 \mathrm{kpc}$ above and below the Galactic plane) in the halo contribution, a non-negligible fraction of CRs still stay in this region and hence the total CR energy budget in the halo is reduced. Given that the average slope of the $\mathrm{CR}$ injection spectral index inferred from gamma-ray emissions of local galaxies is suggested to be $p=2.1-2.2$ (Neronov et al. 2017; Yang et al. 2016), it is clear that the current measurement on IGRB already approaches an interesting level to give a nontrivial constraint on CR luminosity for $D(1 \mathrm{TeV}) \leqslant 10^{30} \mathrm{~cm}^{2} \mathrm{~s}^{-1}$. From Figure 4, we see that for $D(1 \mathrm{TeV}) \leqslant 10^{29} \mathrm{~cm}^{2} \mathrm{~s}^{-1}$, the $\mathrm{CR}$ luminosity can be restricted to be even much smaller than the 
standard Galactic CR luminosity, i.e., $\sim 10^{41} \mathrm{erg} \mathrm{s}^{-1}$, especially in the "no wind" case.

We note that the upper limit for CR luminosity obtained in Figure 4 may be quite conservative, because the IGRB is expected to be dominated by unresolved extragalactic sources (Ajello et al. 2015; Ackermann et al. 2016; Lisanti et al. 2016; see also the analysis in Liu et al. 2016), such as BL Lacs (Di Mauro et al. 2014b) and radio galaxies (Di Mauro et al. 2014a; Hooper et al. 2016). The room left for the halo contribution could be just a small fraction of the value of the IGRB upper limit. For example, if $90 \%$ of the IGRB upper limit at $700 \mathrm{GeV}$ turns out to be contributed by unresolved sources, the allowed Galactic CR luminosity will be ten times smaller than the value obtained in Figure 4 for the same diffusion coefficient. We expect the next-generation very-high-energy gamma-ray detectors, such as CTA, to be able to resolve more extragalactic $\mathrm{TeV}$ gamma-ray sources from the IGRB, providing/allowing a more accurate limit for the halo contribution. If so, the constraint on the Galactic CR injection would become stringent and provide useful clues to the origin of Galactic CRs. For example, provided a smaller $\mathrm{CR}$ luminosity, the required acceleration efficiency of supernova remnants could be lower, and other potential CR accelerators such as OB associations (Cesarsky \& Montmerle 1983; Bykov 2001; Aharonian et al. 2018), pulsars (Bednarek \& Bartosik 2004), and GCs (HESS Collaboration et al. 2016) can possibly account for the majority of Galactic CRs in terms of energy budget.

\section{Discussion}

\subsection{Alternative Ways to Reduce the Sub-TeV Gamma-Ray Flux Produced in the Halo}

A free-escape boundary of CRs has been considered in many previous literature that study the CR transport in the Galaxy. Such a boundary is usually assumed to be located several kiloparsecs above and below the Galactic plane. Once CRs cross the boundary, they will leave the Galaxy and never return, and the CR density is also imposed to be zero at the free-escape boundary (in many numerical studies such as Strong \& Moskalenko 1998; Evoli et al. 2008; Kissmann 2014). The estimated CR luminosity based on this model is usually in the range of $(0.30-3) \times 10^{41} \mathrm{erg} \mathrm{s}^{-1}$. In the previous section, we have shown that in the presence of a turbulent halo, the subTeV IGRB upper limit may imply a smaller Galactic CR luminosity than the conventional value based on the assumption of a free-escape boundary. This is because the physical picture of CR transportation in these two scenarios is different (see Figure 5). CRs that have propagated into the halo that is far away from the Galactic plane may still have a chance to return if the halo is turbulent, especially if there is no large-scale convective Galactic wind or the wind is weak. As a result, the required CR luminosity to maintain the locally observed CR energy density is also smaller than that in the case with a freeescape boundary.

There are also other mechanisms to reduce the theoretical gamma-ray flux at sub-TeV energy, without invoking a small Galactic CR luminosity. An apparent solution is to employ a large diffusion coefficient in the extended halo and a soft injection spectrum (e.g., $p=2.4$ ). As can be seen in Figure 4, such a combination relaxes the constraint on the CR luminosity significantly. On the other hand, IGRB flux at sub-Tev energies actually only constrains the luminosity of

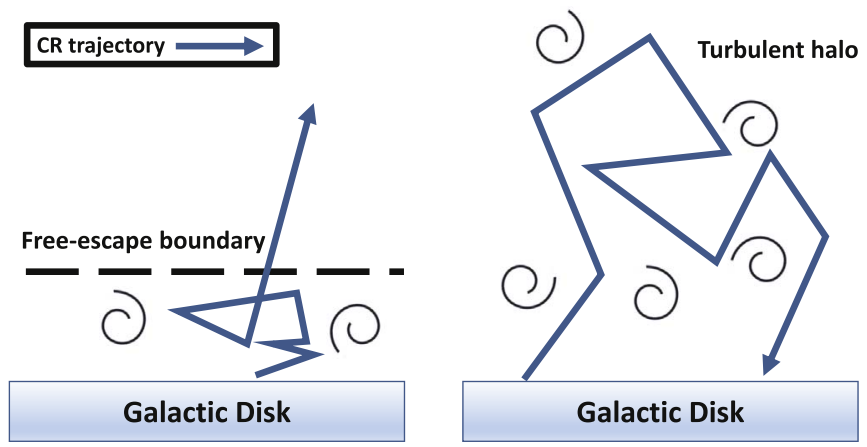

Figure 5. Cartoon illustrating the difference of CR propagation with a freeescape boundary (left) and with a turbulent halo surrounding the Galaxy (right). See Section 4.1 for more discussion.

$\sim 1-10 \mathrm{TeV}$ CRs, which do not necessarily originate from the same sources as $\mathrm{GeV}$ CRs where most of the CR energy resides. There must be fewer TeV CR sources than GeV CR sources since the requirement for accelerating higher energy $\mathrm{CRs}$ is more demanding. It is possible that the measured $\mathrm{TeV}$ CRs are subject to a few nearby sources rather than the sources in the entire Galaxy, such that the total TeV CR luminosity is smaller than the currently inferred value, which is based on the assumption that TeV CR flux is the same in the entire Galactic disk as that measured at Earth. Consequently, the sub-TeV gamma-rays produced in the halo can be reduced effectively. This scenario is implied by the recent discovery of the spectral hardening of Galactic CRs at $\sim 200 \mathrm{GV}$ (e.g., Panov et al. 2009; Adriani et al. 2011; Yoon et al. 2011; Aguilar et al. 2015), since CR spectrum from closer and younger sources tend to be harder because the spectrum is less affected by the energy-dependent diffusion. The anisotropy study on TeV CRs may also support such a scenario (Ahlers 2016).

\subsection{Anisotropy of Gamma-Ray Intensity}

As pointed out in previous studies (Feldmann et al. 2013; Kalashev \& Troitsky 2016), we can expect large-scale anisotropy in the intensity of gamma-rays produced in the halo at different Galactic longitudes $l$ and Galactic latitudes $b$, unlike that which originated from extragalactic sources and is supposed to be roughly isotropic. In particular, due to the offset of Earth from the GC, the gamma-ray intensity should be enhanced toward the GC direction $\left(l=0^{\circ}\right)$ and decreased toward the anti-GC direction $\left(l=180^{\circ}\right)$. We here present the intensity map of $700 \mathrm{GeV}$ gamma-rays produced in the halo by integrating the gamma-ray flux in the line of sight for each $l$ and $b$. As exhibited in Figure 6, the upper panel shows the case with a wind speed of $300 \mathrm{~km} \mathrm{~s}^{-1}$ while the lower panels shows the "no wind" case. In both panels, the injection indexes of CRs $p=2.2$ are adopted, and the all-sky averaged gamma-ray intensity is normalized to the upper limit of the IGRB at the highest energy bin measured by Fermi-LAT. We can see that the gamma-ray intensity varies with $l$ and $b$. The presence of a large-scale Galactic wind with spherical symmetry tends to reduce the anisotropy, because the convection by the wind is isotropic, and more CRs are transported to larger distances in the anti-GC direction. Remarkably, due to a higher CR density and gas density in the inner halo, the gamma-ray emissivity is relatively high, and we can see a bubble-like structure above and below the GC in both cases of $v_{w}=300 \mathrm{~km} \mathrm{~s}^{-1}$ and "no 


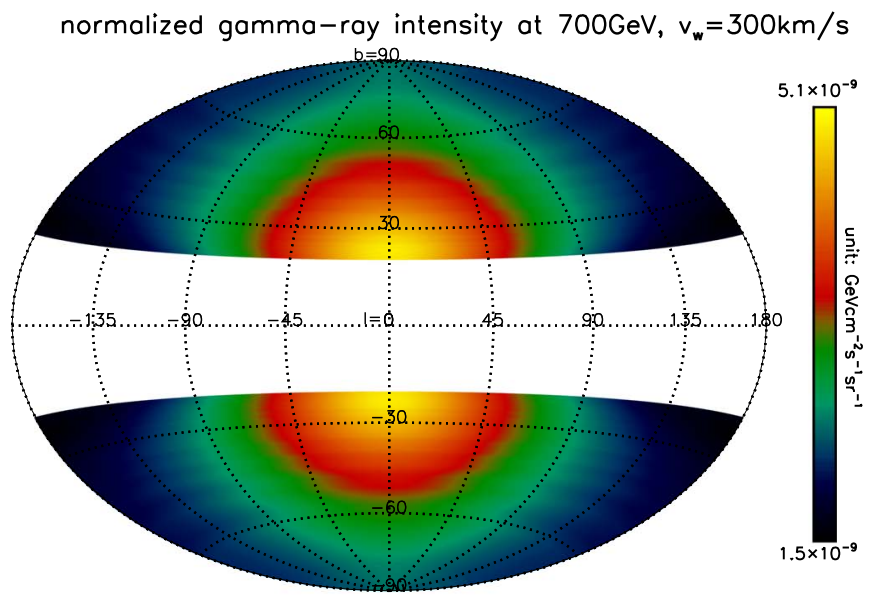

normalized gamma-ray intensity at $700 \mathrm{GeV}$, no wind

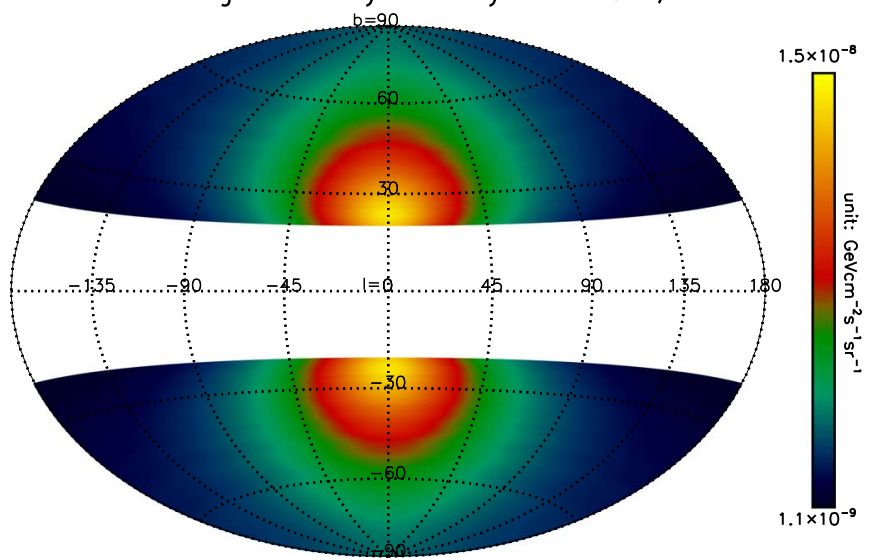

Figure 6. Sky map in Galactic coordinates of high-latitude $\left(|b|>20^{\circ}\right)$ gammaray intensity at $700 \mathrm{GeV}$ produced in the halo. The upper panel shows the case with a large-scale Galactic wind of $v_{w}=300 \mathrm{~km} \mathrm{~s}^{-1}$ while no wind appears in the lower panel. The injection spectral index of CRs is $p=2.2$ in both panels. The all-sky average gamma-ray intensity is normalized to the upper limit of the highest energy bin of the IGRB measured by Fermi-LAT. The color scale is linear. See Section 4.2 for details.

wind." We have also calculated the gamma-ray intensity map employing, instead of a radial wind, a wind perpendicular to the Galactic plane for the inner $15 \mathrm{kpc}$ region, and a similar bubble-like structure appears all the same.

Whether such a structure is related to the Fermi bubble ( $\mathrm{Su}$ et al. 2010; Yang et al. 2014), which is mainly detected at $10-100 \mathrm{GeV}$ with a much higher intensity, remains to be studied in detail. Given the constraint of the IGRB upper limit at $700 \mathrm{GeV}$, the $10 \mathrm{GeV}$ intensity of the bubble-like region in Figure 6 is about one order of magnitude smaller than that of the Fermi bubble. Nevertheless, we note that the real situation is much more complex. For example, the intensity of the bubble-like region can be enhanced if we consider an additional $\mathrm{CR}$ injection from the GC region due to its past activity (Guo \& Mathews 2012; Barkov \& Bosch-Ramon 2014; Crocker et al. 2015), which is inferred from X-ray observations (Revnivtsev et al. 2004; Zhang et al. 2015), and the discovery of a PeV proton accelerator at the GC (HESS Collaboration et al. 2016; Liu et al. 2016; Fujita et al. 2017). Besides, the intensity and morphology of the bubble-like structure can be influenced by the diffusion coefficient or possible outflow in the bubble region, which is not necessarily the same as those in the rest of the halo. Also, $10 \mathrm{GeV}$ gamma-rays are mainly

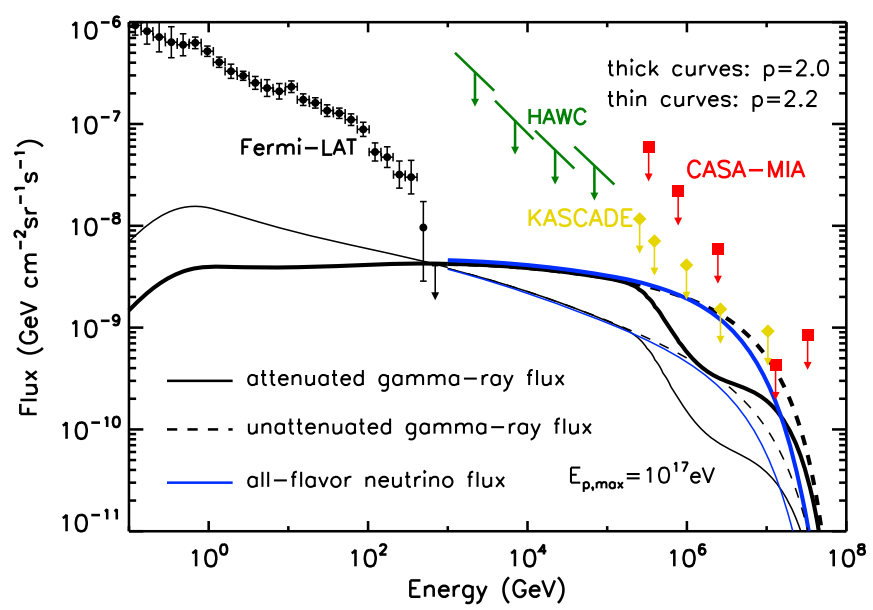

Figure 7. Gamma-ray flux (black curves) and neutrino flux (blue curve) produced in the Galactic halo, assuming maximum CR energy at injection to be $E_{p, \max }=10^{17} \mathrm{eV}$. The gamma-ray fluxes are normalized to the upper limit of the IGRB measured by Fermi-LAT at $700 \mathrm{GeV}$. Thick curves represent the results with $p=2.0$ while thin curves represent the results with $p=2.2$. Black solid curves are the gamma-ray flux after considering the absorption by the $\mathrm{CMB}$, and the black dashed ones are those before the absorption. Black filled circles with error bars represent the IGRB measured by Fermi-LAT (Ackermann et al. 2015), green slashes show the upper limit of diffuse gamma-ray flux from the northern Fermi bubble region measured by HAWC (Abeysekara et al. 2017), yellow squares show the upper limit of the isotropic diffuse gamma-ray flux measured by KASCADE (Schatz et al. 2003), and red diamonds show the upper limit of the isotropic diffuse gamma-ray flux measured by CASA-MIA (Chantell et al. 1997).

produced by $100 \mathrm{GeV}$ protons, at which energy the CR selfregulation via scattering off self-excited Alfvén waves most likely has an influence on the $\mathrm{CR}$ transport and results in a smaller diffusion coefficient than the benchmark one. A detailed study on the Fermi bubble is, however, beyond the scope of this work.

\subsection{Gamma-Ray and Neutrino Production at TeV-PeV}

In the calculation above, we assume a maximum $\mathrm{CR}$ proton energy $E_{p, \max }=10^{15} \mathrm{eV}$ at injection. The maximum CR energy attainable in the Galactic sources could be much higher so that we can expect the gamma-ray spectrum to extend well beyond $\mathrm{TeV}$ energies. High-energy neutrinos can also be produced in the $p p$ collision. In Figure 7, we show the expected gamma-ray flux and neutrino flux produced in the halo by assuming $E_{p, \max }=10^{17} \mathrm{eV}$, which is the suggested maximum proton energy achievable in supernova remnants (Ptuskin \& Zirakashvili 2003), and adopting gas density model A and the fiducial diffusion coefficient. The attenuation of gamma-ray by the CMB is considered, while cosmic infrared background and the infrared radiation from our Galaxy are not important to the attenuation and hence ignored. The gamma-ray flux is normalized to the IGRB upper limit at $700 \mathrm{GeV}$ to show the maximum neutrino flux produced in the halo, so it makes no difference to the resulting gamma-ray/neutrino flux whether or not we consider a Galactic wind. Compared to the constraints from observations of HAWC, CASA-MIA , and KASCADE above $\mathrm{TeV}$, the constraint from Fermi-LAT is the most stringent as long as $p \geqslant 2$. Note that, however, if the maximum proton energy is larger than $10^{18} \mathrm{eV}$, the gamma-ray flux will extend beyond $10^{17} \mathrm{eV}$, at which energy the attenuation due to CMB becomes weak since the attenuation length of $10^{17} \mathrm{eV}$ photon is about $\sim 100 \mathrm{kpc}$, which is comparable to the size of 
the halo. In this case, if the injection spectrum is hard, e.g., $p=2$, the CASA-MIA data may be more constraining than the Fermi-LAT data.

Given the constraint by $\lesssim \mathrm{TeV}$ IGRB, the all-flavor flux of high-energy neutrinos produced in the halo can at most reach the same level as the upper limit of IGRB at $700 \mathrm{GeV}$. Assuming a flavor ratio of 1: 1: 1 among the three flavors after oscillation, we find that the obtained per-flavor neutrino flux can contribute at most about $3 \times 10^{-9} \mathrm{GeV} \mathrm{cm}^{-2} \mathrm{~s}^{-1} \mathrm{sr}^{-1}$ or $(10-30) \%$ of the measured flux by IceCube at $100 \mathrm{TeV}$, i.e., $\simeq(1-3) \times 10^{-8} \mathrm{GeV} \mathrm{cm}^{2} \mathrm{~s}^{-1} \mathrm{sr}^{-1}$ (depending on whether we compare with the high-energy starting events data or the through-going muon data; Aartsen et al. 2015; IceCube Collaboration et al. 2017; Palladino \& Winter 2018), unless the Galactic CRs are injected with a harder spectrum $(p<2)$. This result is consistent with the conclusions in previous literature (e.g., Ahlers \& Murase 2014; Murase et al. 2016). Note that the constraint on the neutrino flux put by the $\lesssim \mathrm{TeV}$ IGRB is weaker for more distant sources (Chang et al. 2016), since the attenuation of $\lesssim \mathrm{TeV}$ gamma-rays by the cosmic infrared background will be more important.

\subsection{Baryon Budget of Hot CGM of our Galaxy}

The cosmic mean baryon fraction is found to be $\approx 16.5 \%$ from the CMB observation with high accuracy (Hinshaw et al. 2013). However, the total observed baryonic mass within the virial radius of the Galaxy is much smaller than the inferred mass from the cosmic mean baryon fraction. This has motivated a great deal of effort to uncover the baryons that are "missing" from the Galaxy (for a review, see Bregman 2007; Tumlinson et al. 2017). There have been predictions that the missing baryons are residing in a hot state in the Galactic halo (White \& Rees 1978; White \& Frenk 1991), which is known as the CGM. While Bregman et al. (2018) suggested that the hot gas does not account for the missing baryons by a factor of 3-10, the total mass of the hot gas in the halo, however, is actually far from settled, since the conclusion suffered from the uncertainties of metallicity (Faerman et al. 2017; Qu \& Bregman 2018), radiative transfer effects (Li \& Bregman 2017), halo rotation (Hodges-Kluck et al. 2016), the size of the halo, and the possible flattening of the gas distribution with respect to the $\beta$ model at the larger radius (Gupta et al. 2012; Faerman et al. 2017). For example, with a flatter density profile at larger radii, Faerman et al. (2017) suggested that the mass of warm/hot gas is as high as $1.2 \times 10^{11} M_{\odot}$, which may account for the missing baryons in the Galaxy, although Bregman et al. (2018) argued that the flattening of $\beta<0.4$ is not consistent with the O VII column observations toward the LMC and SMC.

The gas mass and their distribution in the halo are apparently important to the production of $\gamma$-ray background; therefore, our results may in turn provide an independent constraint on the baryonic component of Galactic gaseous halo, if the properties of Galactic CRs can be fixed. For example, in the fiducial case, we find that the mass of the hot gas halo cannot exceed $\sim 2.6 \times 10^{10} M_{\odot}$, which is only $\sim 11 \%$ of the missing baryon mass in the Galaxy given a total mass of $\sim 1.4 \times 10^{12} M_{\odot}$ (Watkins et al. 2018) for the Galaxy, in order not to overshoot the IGRB upper limit at $\sim 700 \mathrm{GeV}$. We note that the result is subject to the uncertainty of various model parameters. We then explore the influences of the diffusion coefficient, the wind speed, and the injection CR spectral index on the result in

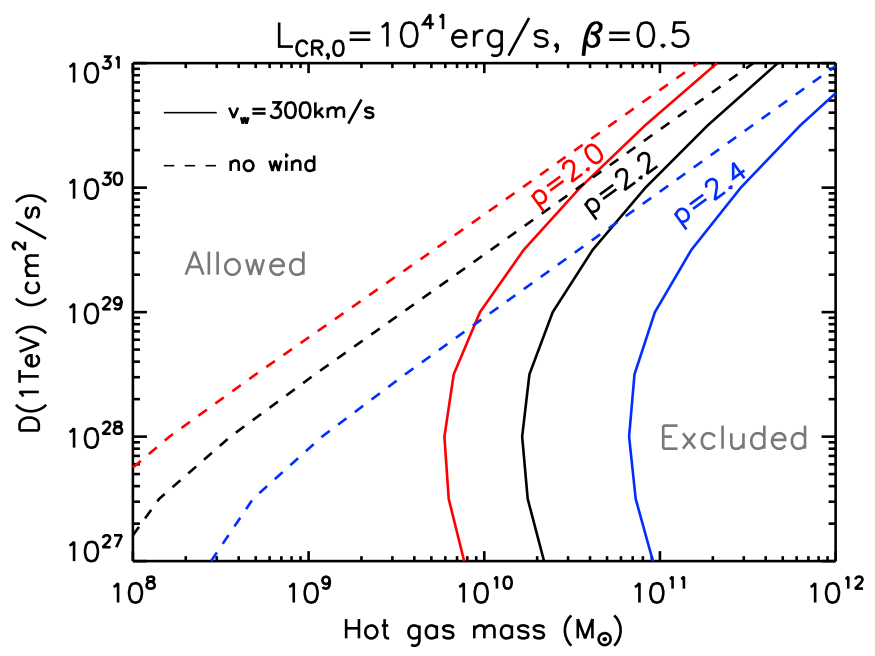

Figure 8. The maximum hot gas mass in the halo that will not overproduce the IGRB flux at $700 \mathrm{GeV}$. Solid curves show the results with a wind speed $v_{w}=300 \mathrm{~km} \mathrm{~s}^{-1}$ while dashed curves show the results without wind. Red, black, and blue curves represent the results with $p=2.0,2.2$, and 2.4, respectively. In the figure, the present-time CR luminosity is fixed at $10^{41} \mathrm{erg} \mathrm{s}^{-1}$, and $\beta=0.5$ is adopted for the gas density profile in the halo.

Figure 8. The CR luminosity in the calculation is fixed at $10^{41} \mathrm{erg} \mathrm{s}^{-1}$, and we do not explore the dependence on it since the upper limit of the gas mass is simply proportional to $L_{\mathrm{CR}, 0}^{-1}$. In the plot, we fix $\beta$ at 0.5 and adjust the amplitude in the gas density profile $\left(n_{0}\right)$ to make the predicted gamma-ray flux at $700 \mathrm{GeV}$ equal to the measured upper limit. The maximum gas mass can then be calculated by integrating the gas density profile over the entire volume within the virial radius. Similar to Figure 4, the region to the left region of a curve is the allowed parameter space in the corresponding condition of the curve. Considering that the Galactic halo only contributes a fraction of the total IGRB, the constraint on the halo gas mass will be even stronger if a better estimate of the extragalactic contribution and/or a more accurate upper limit of the IGRB at $\sim 700 \mathrm{GeV}$ are obtained. If the total gas mass in the halo is fixed, a smaller $\beta$ leads to a lower gas density than the case of a large $\beta$ in the inner halo where the CR density is relatively high and the distance to Earth is small. As a result, the expected gamma-ray flux from the halo will be smaller, allowing a larger amount of gas in the halo. Hence, all of the curves in Figure 8 will shift to the right.

\section{Conclusion}

To summarize, we calculated the distribution of Galactic CRs in the extended halo after they leave the Galaxy and the gamma-ray production by these CRs via the $p p$ collision with the gas in the halo. Given a total gas mass of $10^{10}-10^{11} M_{\odot}$ in the halo, we found that the current measurement of the IGRB at $\lesssim \mathrm{TeV}$ by Fermi-LAT already approaches a level that may provide an independent nontrivial constraint on the luminosity of Galactic CRs. In our fiducial case, the present-time Galactic $\mathrm{CR}$ luminosity is required to be smaller than $10^{41} \mathrm{erg} \mathrm{s}^{-1}$ in order not to overshoot the measured IGRB upper limit with the predicted gamma-ray flux produced in the halo. Because the IGRB is expected to be dominated by extragalactic sources, future observations on the extragalactic gamma-ray sky may further shrink the room left for the Galactic halo contribution and provide an even stronger constraint on the Galactic CR luminosity and their origin. A small Galactic CR luminosity is 
consistent with the presence of an extended turbulent halo surrounding the Galaxy, since in this scenario CRs that leave the Galaxy will have a chance of returning. Our constraint on CR luminosity is influenced by various model parameters. We found that a large diffusion coefficient in the extended halo (i.e., $D>10^{29} \mathrm{~cm}^{2} \mathrm{~s}^{-1}$ at $E=1 \mathrm{TeV}$ ) or a soft injection spectrum of CRs (i.e., $p>2.2$ ) can reduce the predicted gamma-ray flux, relaxing the constraint on the CR luminosity. An alternative possible scenario that can reduce the predicted sub-TeV gamma-ray flux is the presence of a local overdensity of a TeV CR source.

A few more implications of our results may be worth noting. First, the interaction between CRs injected from the Galactic plane and gas in the halo can produce a bubble-like structure in gamma-rays above and below the Galactic plane. Given the constraint from the IGRB upper limit at $\lesssim \mathrm{TeV}$, the intensity of the bubble-like structure is much smaller than that of the Fermi bubble. Additional CR injection from the GC and a distinct environment in the region (e.g., a smaller diffusion coefficient or a higher gas density) from the rest of the halo may help explain the Fermi bubble in this scenario. Second, due to the constraints by the IGRB upper limit, high-energy neutrinos produced in the halo can reach at most (10-30)\% of the measured flux by IceCube above $100 \mathrm{TeV}$. Last, if the properties of the Galactic CRs can be fixed, the IGRB upper limit may also be useful to constrain the baryon budget of the hot gas in the halo. For example, our fiducial model constrains the hot gas mass in the halo to be lower than $2.6 \times 10^{10} M_{\odot}$. However, this value is subject to the adopted CR spectral index at injection, diffusion coefficient, as well as the gas profile in the halo. For $p=2.4, D(1 \mathrm{TeV})=10^{29} \mathrm{~cm}^{2} \mathrm{~s}^{-1}$, and $\beta=0.5$, the maximal mass is $10^{11} M_{\odot}$.

We thank Felix Aharonian and the anonymous referee for invaluable comments, and thank Martin Pohl, Christoph Pfrommer, and Susumu Inoue for helpful discussions. This work is partially supported by the National Key Research and Development Program of China under grant 2018YFA0404203 and NSFC grants 11625312 and 11851304.

\section{ORCID iDs}

Ruo-Yu Liu (i) https://orcid.org/0000-0003-1576-0961 Huirong Yan (10 https://orcid.org/0000-0003-2560-8066 Hui Li (1) https://orcid.org/0000-0002-1253-2763

\section{References}

Aartsen, M. G., Abraham, K., Ackermann, M., et al. 2015, ApJ, 809, 98 IceCube Collaboration, Aartsen, M. G., Ackermann, M., et al. 2017, arXiv: 1710.01191

Abdo, A. A., Ackermann, M., Ajello, M., et al. 2009, PhRvL, 103, 251101 Abdo, A. A., Ackermann, M., Ajello, M., et al. 2010, PhRvL, 104, 101101 Abeysekara, A. U., Albert, A., Alfaro, R., et al. 2017, ApJ, 842, 85 Ackermann, M., Ajello, M., Albert, A., et al. 2015, ApJ, 799, 86 Ackermann, M., Ajello, M., Albert, A., et al. 2016, PhRvL, 116, 151105 Ackermann, M., Ajello, M., Allafort, A., et al. 2013, Sci, 339, 807 Adriani, O., Barbarino, G. C., Bazilevskaya, G. A., et al. 2011, Sci, 332, 69 Aguilar, M., Aisa, D., Alpat, B., et al. 2015, PhRvL, 115, 211101 Aguilar, M., Ali Cavasonza, L., Ambrosi, G., et al. 2016, PhRvL, 117, 231102 Aharonian, F., Yang, R., \& de Oña Wilhelmi, E. 2018, arXiv:1804.02331 Ahlers, M. 2016, PhRvL, 117, 151103

Ahlers, M., \& Murase, K. 2014, PhRvD, 90, 023010

Ajello, M., Gasparrini, D., Sánchez-Conde, M., et al. 2015, ApJL, 800, L27

Barkov, M. V., \& Bosch-Ramon, V. 2014, A\&A, 565, A65
Bednarek, W., \& Bartosik, M. 2004, A\&A, 423, 405

Behroozi, P. S., Wechsler, R. H., \& Conroy, C. 2013, ApJ, 770, 57

Berezinskii, V. S., Bulanov, S. V., Dogiel, V. A., \& Ptuskin, V. S. 1990, in Astrophysics of Cosmic Rays, ed. V. L. Ginzburg (Amsterdam: North Holland)

Berezinsky, V., \& Gazizov, A. Z. 2006, ApJ, 643, 8

Bregman, J. N. 2007, ARA\&A, 45, 221

Bregman, J. N., Anderson, M. E., Miller, M. J., et al. 2018, ApJ, 862, 3

Breitschwerdt, D., McKenzie, J. F., \& Voelk, H. J. 1991, A\&A, 245, 79

Bykov, A. M. 2001, SSRv, 99, 317

Cen, R. 2012, ApJ, 753, 17

Cen, R., \& Ostriker, J. P. 2006, ApJ, 650, 560

Cesarsky, C. J., \& Montmerle, T. 1983, SSRv, 36, 173

Chang, X.-C., Liu, R.-Y., \& Wang, X.-Y. 2016, ApJ, 825, 148

Chantell, M. C., Covault, C. E., Cronin, J. W., et al. 1997, PhRvL, 79, 1805

Coppi, P. S., \& Aharonian, F. A. 1997, ApJL, 487, L9

Crocker, R. M., Bicknell, G. V., Taylor, A. M., \& Carretti, E. 2015, ApJ, 808, 107

De Paolis, F., Ingrosso, G., Jetzer, P., \& Roncadelli, M. 2000, NJPh, 2, 12

Di Mauro, M., Calore, F., Donato, F., Ajello, M., \& Latronico, L. 2014a, ApJ, 780, 161

Di Mauro, M., Donato, F., Lamanna, G., Sanchez, D. A., \& Serpico, P. D. 2014b, ApJ, 786, 129

Dogiel, V. A., Schönfelder, V., \& Strong, A. W. 2002, ApJL, 572, L157

Drury, L. O., Markiewicz, W. J., \& Voelk, H. J. 1989, A\&A, 225, 179

Dubois, Y., \& Teyssier, R. 2008, A\&A, 477, 79

Evoli, C., Gaggero, D., Grasso, D., \& Maccione, L. 2008, JCAP, 10, 018

Faerman, Y., Sternberg, A., \& McKee, C. F. 2017, ApJ, 835, 52

Fang, T., Buote, D., Bullock, J., \& Ma, R. 2015, ApJS, 217, 21

Farmer, A. J., \& Goldreich, P. 2004, ApJ, 604, 671

Feldmann, R., Hooper, D., \& Gnedin, N. Y. 2013, ApJ, 763, 21

Fichtel, C. E., Hartman, R. C., Kniffen, D. A., et al. 1975, ApJ, 198, 163

Fichtel, C. E., Simpson, G. A., \& Thompson, D. J. 1978, ApJ, 222, 833

Fielding, D., Quataert, E., Martizzi, D., \& Faucher-Giguère, C.-A. 2017, MNRAS, 470, L39

Fletcher, R. S., Gaisser, T. K., Lipari, P., \& Stanev, T. 1994, PhRvD, 50, 5710

Fox, A. J., Wakker, B. P., Savage, B. D., et al. 2005, ApJ, 630, 332

Fujita, Y., Murase, K., \& Kimura, S. S. 2017, JCAP, 4, 037

Ginzburg, V. L., \& Syrovatskii, S. I. 1964, in The Origin of Cosmic Rays, ed. D. ter Haar (New York: Macmillan)

Grcevich, J., \& Putman, M. E. 2009, ApJ, 696, 385

Guo, F., \& Mathews, W. G. 2012, ApJ, 756, 181

Gupta, A., Mathur, S., Krongold, Y., Nicastro, F., \& Galeazzi, M. 2012, ApJL, 756, L8

Hanasz, M., Lesch, H., Naab, T., et al. 2013, ApJL, 777, L38

Heesen, V., Krause, M., Beck, R., et al. 2018, MNRAS, 476, 158

Henley, D. B., \& Shelton, R. L. 2012, ApJS, 202, 14

Henley, D. B., \& Shelton, R. L. 2013, ApJ, 773, 92

HESS Collaboration, Abramowski, A., Aharonian, F., et al. 2016, Natur, 531,476

Hinshaw, G., Larson, D., Komatsu, E., et al. 2013, ApJS, 208, 19

Hodges-Kluck, E. J., Miller, M. J., \& Bregman, J. N. 2016, ApJ, 822, 21

Hooper, D., Linden, T., \& Lopez, A. 2016, JCAP, 8, 019

Kalashev, O., \& Troitsky, S. 2016, PhRvD, 94, 063013

Kelner, S. R., Aharonian, F. A., \& Bugayov, V. V. 2006, PhRvD, 74, 034018 Kissmann, R. 2014, APh, 55, 37

Kulsrud, R., \& Pearce, W. P. 1969, ApJ, 156, 445

Li, Y., \& Bregman, J. 2017, ApJ, 849, 105

Lisanti, M., Mishra-Sharma, S., Necib, L., \& Safdi, B. R. 2016, ApJ, 832, 117

Liu, R.-Y., Taylor, A. M., Wang, X.-Y., \& Aharonian, F. A. 2016, PhRvD, 94, 043008

Miller, M. J., \& Bregman, J. N. 2013, ApJ, 770, 118

Miller, M. J., \& Bregman, J. N. 2015, ApJ, 800, 14

Mulcahy, D. D., Fletcher, A., Beck, R., Mitra, D., \& Scaife, A. M. M. 2016, A\&A, 592, A123

Murase, K., Guetta, D., \& Ahlers, M. 2016, PhRvL, 116, 071101

Muratov, A. L., Kereš, D., Faucher-Giguère, C.-A., et al. 2015, MNRAS, 454, 2691

Neronov, A., Malyshev, D., \& Semikoz, D. V. 2017, A\&A, 606, A22

Nicastro, F., Zezas, A., Drake, J., et al. 2002, ApJ, 573, 157

O’C. Drury, L. 2017, arXiv:1708.08858

Orlando, E., \& Strong, A. 2013, MNRAS, 436, 2127

Palladino, A., \& Winter, W. 2018, A\&A, 651, 168

Panov, A. D., Adams, J. H., Ahn, H. S., et al. 2009, BRASP, 73, 564

Ptuskin, V. S., \& Zirakashvili, V. N. 2003, A\&A, 403, 1 
Putman, M. E., Saul, D. R., \& Mets, E. 2011, MNRAS, 418, 1575

Qu, Z., \& Bregman, J. N. 2018, ApJ, 856, 5

Rasmussen, A., Kahn, S. M., \& Paerels, F. 2003, in The IGM/Galaxy

Connection. The Distribution of Baryons at $\mathrm{z}=0,281$ ed. J. L. Rosenberg \& M. E. Putman (Dordrecht: Springer), 109

Recchia, S., Blasi, P., \& Morlino, G. 2016, MNRAS, 462, 4227

Revnivtsev, M. G., Churazov, E. M., Sazonov, S. Y., et al. 2004, A\&A, 425, L49

Salem, M., \& Bryan, G. L. 2014, MNRAS, 437, 3312

Sarkar, K. C., Nath, B. B., Sharma, P., \& Shchekinov, Y. 2015, MNRAS, 448, 328

Schatz, G., Feßler, F., Antoni, T., et al. 2003, ICRC, 4, 2293

Skilling, J. 1971, ApJ, 170, 265

Snaith, O., Haywood, M., Di Matteo, P., et al. 2015, A\&A, 578, A87

Sreekumar, P., Bertsch, D. L., Dingus, B. L., et al. 1998, ApJ, 494, 523

Stanimirović, S., Dickey, J. M., Krčo, M., \& Brooks, A. M. 2002, ApJ, 576,773

Stecker, F. W., \& Jones, F. C. 1977, ApJ, 217, 843

Strong, A. W., \& Moskalenko, I. V. 1998, ApJ, 509, 212

Strong, A. W., Moskalenko, I. V., \& Reimer, O. 2004, ApJ, 613, 962

Strong, A. W., Porter, T. A., Digel, S. W., et al. 2010, ApJL, 722, L58
Su, M., Slatyer, T. R., \& Finkbeiner, D. P. 2010, ApJ, 724, 1044

Taylor, A. M., Gabici, S., \& Aharonian, F. 2014, PhRvD, 89, 103003

Taylor, A. M., \& Giacinti, G. 2017, PhRvD, 95, 023001

Toft, S., Rasmussen, J., Sommer-Larsen, J., \& Pedersen, K. 2002, MNRAS, 335,799

Tumlinson, J., Peeples, M. S., \& Werk, J. K. 2017, ARA\&A, 55, 389

Vogelsberger, M., Genel, S., Sijacki, D., et al. 2013, MNRAS, 436, 3031

Watkins, L. L., van der Marel, R. P., Sohn, S. T., \& Evans, N. W. 2018 , arXiv: 1804.11348

White, S. D. M., \& Frenk, C. S. 1991, ApJ, 379, 52

White, S. D. M., \& Rees, M. J. 1978, MNRAS, 183, 341

Yan, H., \& Lazarian, A. 2002, PhRvL, 89, 281102

Yan, H., \& Lazarian, A. 2008, ApJ, 673, 942

Yang, R., Aharonian, F., \& Evoli, C. 2016, PhRvD, 93, 123007

Yang, R.-Z., Aharonian, F., \& Crocker, R. 2014, A\&A, 567, A19

Yoon, Y. S., Ahn, H. S., Allison, P. S., et al. 2011, ApJ, 728, 122

Zhang, S., Hailey, C. J., Mori, K., et al. 2015, ApJ, 815, 132

Zheng, Y., Peek, J. E. G., Putman, M. E., \& Werk, J. K. 2017, arXiv:1710. 10703

Zirakashvili, V. N., Breitschwerdt, D., Ptuskin, V. S., \& Voelk, H. J. 1996, A\&A, 311, 113 